\title{
Highly Robust and Self-Powered Electronic Skin Based on Tough Conductive Self-Healing Elastomer
}

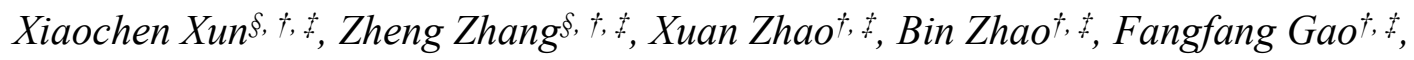
Zhuo Kang ${ }^{\dagger, *}$, Qingliang Liao* $* *$ and Yue Zhang*,$+ \neq$

$\dagger$ Beijing Advanced Innovation Center for Materials Genome Engineering, Beijing Key Laboratory for Advanced Energy Materials and Technologies, University of Science and Technology Beijing, Beijing 100083, People's Republic of China

$¥$ State Key Laboratory for Advanced Metals and Materials, School of Materials

Science and Engineering, University of Science and Technology Beijing, Beijing 100083, People's Republic of China

*E-mail: liao@ustb.edu.cn (Qingliang Liao), yuezhang@ustb.edu.cn (Yue Zhang)

$\S$ These authors contributed equally to this work. 
Synthesis of the self-healing crosslinked insulated PUE triboelectric layer: Poly (propylene glycol), tolylene 2, 4-diisocyanate terminated, $\mathrm{Mw}=2300(0.35 \mathrm{~g}, 0.15$ mmol) was dissolved in $1 \mathrm{~mL}$ tetrahydrofuran (THF) in a $20 \mathrm{~mL}$ glass sample bottle under magnetic stirring. 4-Aminophenyl disulfide (AFD, $33 \mathrm{mg}, 0.133 \mathrm{mmol}$ ) was added and constantly stirred for $15 \mathrm{~min}$. The viscous mixture was transferred to a Teflon mold and degassed under vacuum for $15 \mathrm{~min}$ to completely remove bubbles and solvent. Finally, the degassed viscous liquid was cured for $48 \mathrm{~h}$ at $75^{\circ} \mathrm{C}$ under vacuum. The product was a yellowish transparent elastomer.

Synthesis of the self-healing crosslinked conductive PUE electrode: $85 \mathrm{mg}$ Carbon black was dissolved in $2 \mathrm{~mL}$ THF in a $20 \mathrm{~mL}$ glass sample bottle under magnetic stirring. 0.35g Poly (propylene glycol), tolylene 2, 4-diisocyanate terminated was dissolved in $1 \mathrm{~mL}$ THF in another $20 \mathrm{~mL}$ glass sample bottle under magnetic stirring. Then mix the two solutions and continue to magnetic stir $12 \mathrm{~h}$. Finally, 33mg AFD was added and constantly stirred for $15 \mathrm{~min}$. The viscous mixture was transferred to a Teflon mold and degassed under vacuum for 15 min to completely remove bubbles and solvent. Finally, the degassed viscous liquid was cured for $48 \mathrm{~h}$ at $75^{\circ} \mathrm{C}$ under vacuum. The product was a black elastomer.

After healing, the samples were retested.

\section{Healing efficiency $(\eta)$ was quantitatively calculated by the following equation:}

$$
\text { Healing efficiency }(\eta)=\varepsilon_{\text {heal }} / \varepsilon_{\text {ori }} \times 100 \%(\mathrm{~S} 1)
$$

where $\varepsilon_{\text {heal }}$ is the fracture deformation or response current for healed specimens, and $\varepsilon_{\text {ori }}$ is the fracture deformation or response current for the virgin specimens. 
Table S1. The Young's modulus, stretchability, toughness, resistance, and self-healing efficiency of stretchability for insulated PUE and conductive PUE film with different contents .

\begin{tabular}{|c|c|c|c|c|c|}
\hline Content & $\begin{array}{c}\text { Young's } \\
\text { Modulus }\end{array}$ & Stretchability & Toughness & Resistance & $\begin{array}{c}\text { Self-healing efficiency } \\
\text { of stretchability } \\
\text { (R.T., 24h) }\end{array}$ \\
\hline PUE & $1.6 \mathrm{MPa}$ & $700 \%$ & $32.8 \mathrm{MJ} / \mathrm{m}^{3}$ & $\infty$ & $95 \%$ \\
\hline $15 \mathrm{wt} \% \mathrm{CB}-\mathrm{PUE}$ & $3.2 \mathrm{MPa}$ & $500 \%$ & $35.9 \mathrm{MJ} / \mathrm{m}^{3}$ & $20 \mathrm{M} \Omega$ & $85 \%$ \\
\hline $25 \mathrm{wt} \% \mathrm{CB}-\mathrm{PUE}$ & $4.5 \mathrm{MPa}$ & $300 \%$ & $18.1 \mathrm{MJ} / \mathrm{m}^{3}$ & $2 \mathrm{~K} \Omega$ & $70 \%$ \\
\hline $40 \mathrm{wt} \% \mathrm{CB}-\mathrm{PUE}$ & $10.2 \mathrm{MPa}$ & $100 \%$ & $5.0 \mathrm{MJ} / \mathrm{m}^{3}$ & $500 \Omega$ & $40 \%$ \\
\hline
\end{tabular}

Table S2. Some recent important works about self-healing and self-powered E-skin.

(the italics bold represents the self-healing components).

\begin{tabular}{|c|c|c|c|c|c|c|c|}
\hline Year & Devices (material $1 /$ material $_{2} /$ material $_{3}$ ) & Stretchability & $\begin{array}{l}\text { Modulus } \\
\text { matching }\end{array}$ & $\begin{array}{l}\text { Mechanosensati } \\
\text { on stability }\end{array}$ & $\begin{array}{c}\text { Fully self- } \\
\text { healing } \\
\text { ability }\end{array}$ & Journal & Ref. \\
\hline 2015 & Acrylic/Al/PU/Al & - & $\begin{array}{l}\text { High modulus } \\
\text { mismatch }\end{array}$ & - & - & $\begin{array}{c}\text { Energy } \\
\text { Environ. } \\
\text { Sci. }\end{array}$ & 1 \\
\hline 2017 & PDMS-PU/Magnetic/Ni /PDMS-PU & - & $\begin{array}{l}\text { High modulus } \\
\text { mismatch }\end{array}$ & - & - & $\begin{array}{l}\text { Nano } \\
\text { Energy }\end{array}$ & 2 \\
\hline 2017 & $\mathrm{Al} / \mathrm{PFA} / \boldsymbol{A g} \boldsymbol{N} W \mathrm{~s}-\boldsymbol{P D O} \mathbf{3} / \mathrm{PEN}$ & - & $\begin{array}{l}\text { High modulus } \\
\text { mismatch }\end{array}$ & - & - & $\begin{array}{l}\text { Nano } \\
\text { Energy }\end{array}$ & 3 \\
\hline 2017 & VHB/Hydrogel/Silicone & $800 \%$ & $\begin{array}{l}\text { Low modulus } \\
\text { mismatch }\end{array}$ & $\begin{array}{l}\text { increasing with } \\
\text { deformation }\end{array}$ & - & $\begin{array}{l}\text { Adv. } \\
\text { Mater. }\end{array}$ & 4 \\
\hline 2018 & PDMS/Epoxy/CNT-Epoxy & - & $\begin{array}{l}\text { High modulus } \\
\text { mismatch }\end{array}$ & - & - & $\begin{array}{l}\text { Nano } \\
\text { Energy }\end{array}$ & 5 \\
\hline 2018 & $A g N W \mathrm{~s} / T P U / g a p / P D M S / A g N W s$ & - & $\begin{array}{l}\text { High modulus } \\
\text { mismatch }\end{array}$ & - & $\begin{array}{l}\text { NIR light } \\
\text { irradiation }\end{array}$ & $\begin{array}{l}\text { RSC } \\
\text { Adv. }\end{array}$ & 6 \\
\hline 2018 & $\boldsymbol{H}$-PDMS/AgNWs-PEDOT(Buckling)/H-PDMS & $350 \%$ & $\begin{array}{l}\text { High modulus } \\
\text { mismatch }\end{array}$ & $\begin{array}{c}<40 \% \\
\text { deformation }\end{array}$ & - & $\begin{array}{l}\text { ACS } \\
\text { Nano }\end{array}$ & 7 \\
\hline 2018 & Vitrimer/AgNWs/Vitrimer & $110 \%$ & $\begin{array}{l}\text { High modulus } \\
\text { mismatch }\end{array}$ & $\begin{array}{c}<10 \% \\
\text { deformation }\end{array}$ & $65^{\circ} \mathrm{C}, 4 \mathrm{~h}$ & $\begin{array}{l}\text { Adv. } \\
\text { Mater. }\end{array}$ & 8 \\
\hline 2019 & Putty/CNT-Putty & - & $\begin{array}{l}\text { Low modulus } \\
\text { mismatch }\end{array}$ & - & $\mathrm{RT}, 20 \mathrm{~min}$ & $\begin{array}{l}\text { ACS } \\
\text { Nano }\end{array}$ & 9 \\
\hline \multirow[t]{2}{*}{2019} & HTS-PDMS/HTS-c-Hydrogel & $250 \%$ & $\begin{array}{l}\text { Low modulus } \\
\text { mismatch }\end{array}$ & $\begin{array}{l}\text { Increasing with } \\
\text { deformation }\end{array}$ & - & $\begin{array}{l}\text { Adv. } \\
\text { Funct. } \\
\text { Mater. }\end{array}$ & 10 \\
\hline & $P U E / C B-P U E$ & $350 \%$ & $\begin{array}{l}\text { Low modulus } \\
\text { mismatch }\end{array}$ & $\begin{array}{c}>50 \% \\
\text { deformation }\end{array}$ & $75^{\circ} \mathrm{C}, 24 \mathrm{~h}$ & & $\begin{array}{l}\text { This } \\
\text { work }\end{array}$ \\
\hline
\end{tabular}

\section{References}

(1) Lee, J.; Hinchet, R.; Kim, S.; Kim, S.; Kim, S. Shape Memory Polymer-Based Self-

Healing Triboelectric Nanogenerator. Energy Environ. Sci. 2015, 8, 3605-3613.

(2) Xu, W.; Huang, L.; Hao, J. Fully Self-Healing and Shape-Tailorable Triboelectric

Nanogenerators Based on Healable Polymer and Magnetic-Assisted Electrode. Nano 
Energy 2017, 40, 399-407.

(3) Park, J.; Park, K.; Jiang, T.; Sun, Q.; Huh, J.; Wang, Z.; Lee, S.; Cho, J. LightTransformable and -Healable Triboelectric Nanogenerators. Nano Energy 2017, 38, $412-418$.

(4) Parida, K.; Kumar, V.; Wang, J.; Bhavanasi, V.; Bendi, R.; Lee, P. Highly Transparent, Stretchable, and Self-Healing Ionic-Skin Triboelectric Nanogenerators for Energy Harvesting and Touch Applications. Adv. Mater. 2017, 29, 1702181.

(5) Guan, Q.; Dai, Y.; Yang, Y.; Bi, X.; Wen, Z.; Pan, Y. Near-Infrared Irradiation Induced Remote and Efficient Self-Healable Triboelectric Nanogenerator for Potential Implantable Electronics. Nano Energy 2018, 51, 333-339.

(6) Niu, H.; Du, X.; Zhao, S.; Yuan, Z.; Zhang, X.; Cao, Ran.; Yin, Y.; Zhang, C.; Zhou, T.; Li, C. Polymer Nanocomposite-Enabled High-Performance Triboelectric Nanogenerator with Self-Healing Capability. RSC Adv. 2018, 8, 30661.

(7) Sun, J.; Pu, X.; Liu, M.; Yu, A.; Du, C.; Zhai, J.; Hu, W.; Wang, Z. Self-Healable, Stretchable, Transparent Triboelectric Nanogenerators as Soft Power Sources. ACS Nano 2018, 12, 6147-6155.

(8) Deng, J.; Kuang, X.; Liu, R.; Ding, W.; Wang, A.; Lai, Y.; Dong, K.; Wen, Z.; Wang, Y.; Wang, L.; Qi, H.; Zhang, T.; Wang, Z. Vitrimer Elastomer-Based Jigsaw Puzzle-Like Healable Triboelectric Nanogenerator for Self-Powered Wearable Electronics. Adv. Mater. 2018, 30, 1705918.

(9) Chen, Y.; Pu, X.; Liu, M.; Kuang, S.; Zhang, P.; Hua, Q.; Cong, Z.; Guo, W.; Hu, W.; Wang, Z. Shape-Adaptive, Self-Healable Triboelectric Nanogenerator with 
Enhanced Performances by Soft Solid-Solid Contact Electrification. ACS nano 2019, 13, 8936-8945.

(10)Lai, Y.; Wu, H.; Lin, H.; Chang, C.; Chou, H.; Hsiao, Y.; Wu, Y. Entirely, Intrinsically, and Autonomously Self - Healable, Highly Transparent, and Superstretchable Triboelectric Nanogenerator for Personal Power Sources and SelfPowered Electronic Skins. Adv. Funct. Mater. 2019, 1904626.

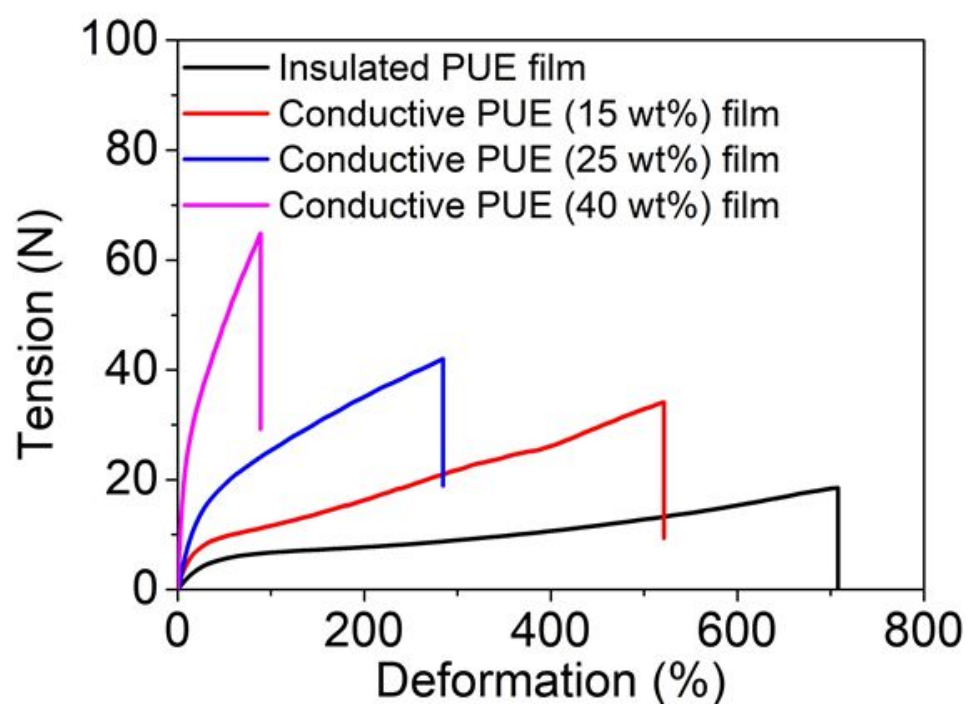

Figure S1. The uniaxial tension-deformation curve of insulated PUE film and conductive PUE film with different doping content of CB obtained by tensile test.

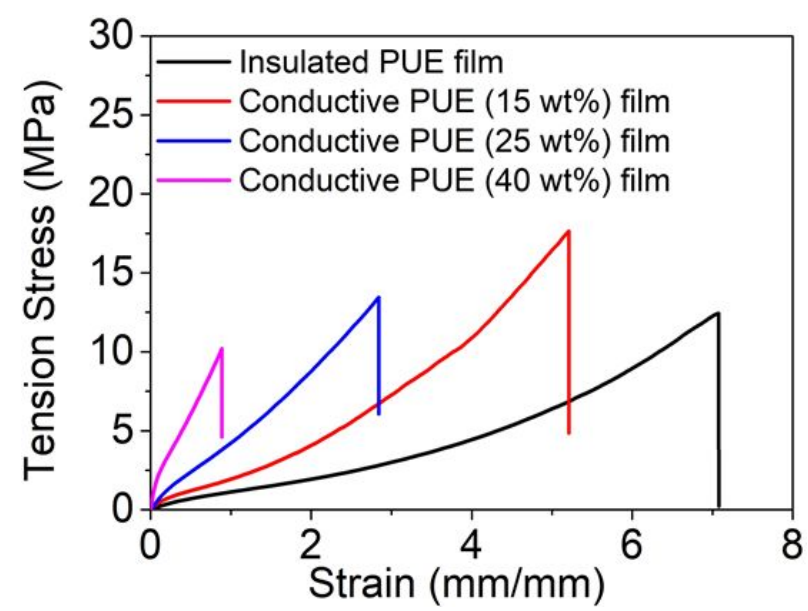

Figure S2. The stress-strain curve of the insulated PUE film and conductive PUE film with different doping content of $\mathrm{CB}$ obtained by tensile test. 


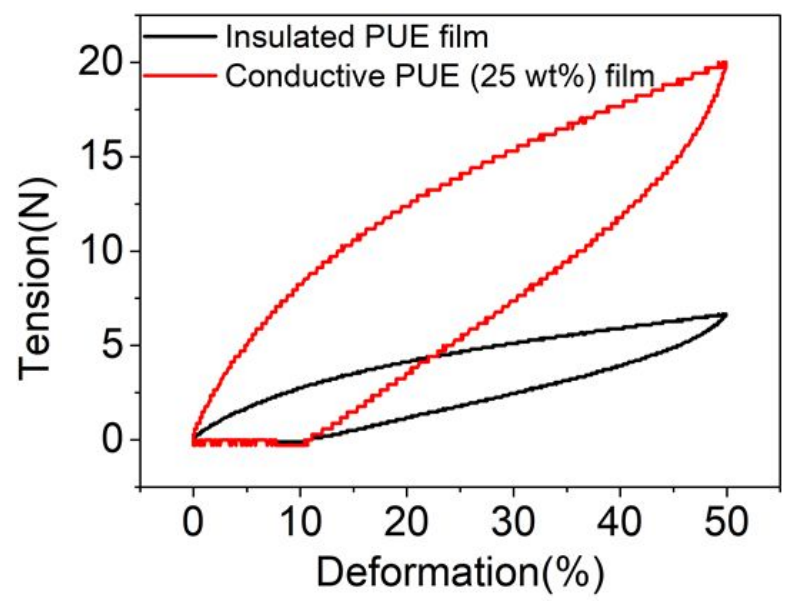

Figure S3. The cycle tension-deformation curves of the insulated PUE film and conductive PUE film obtained by cycle tensile test.

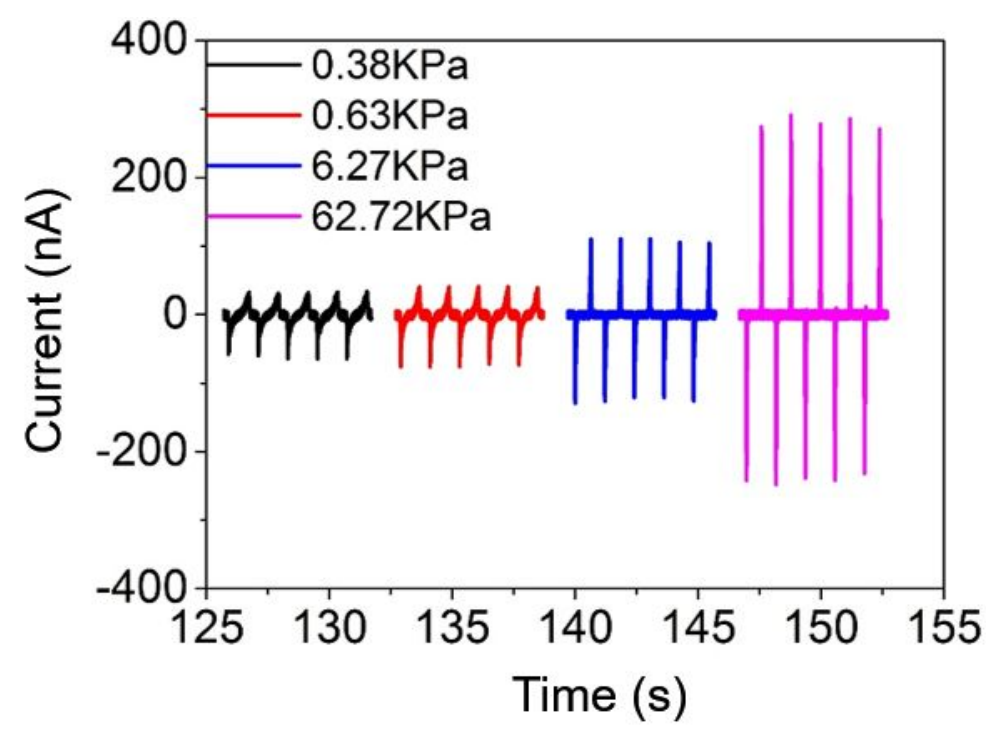

Figure S4. Representative output current profiles of the developed HRSE-skin at 0.38 $\mathrm{KPa}, 0.63 \mathrm{KPa}, 6.27 \mathrm{KPa}, 62.72 \mathrm{KPa}$ pressures.

a

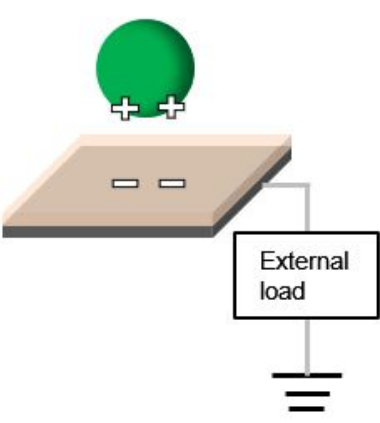

b

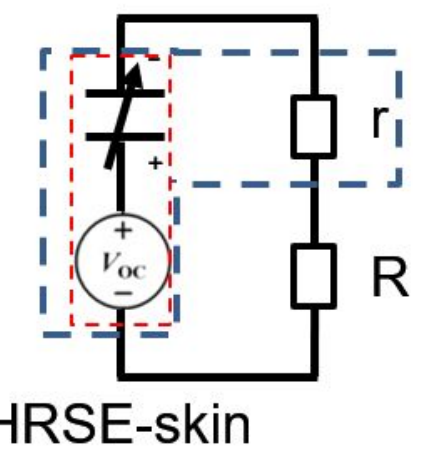


Figure S5. (a) Schematic diagram of an HRSE-skin in series with external loads. (b) Equivalent circuit diagram of a series connection of the HRSE-skin and external Load, the blue line represents the HRSE-skin, the red line represents the inherent impedance of the HRSE-skin, $r$ represents electrode resistance of the HRSE-skin, R represents load resistance.
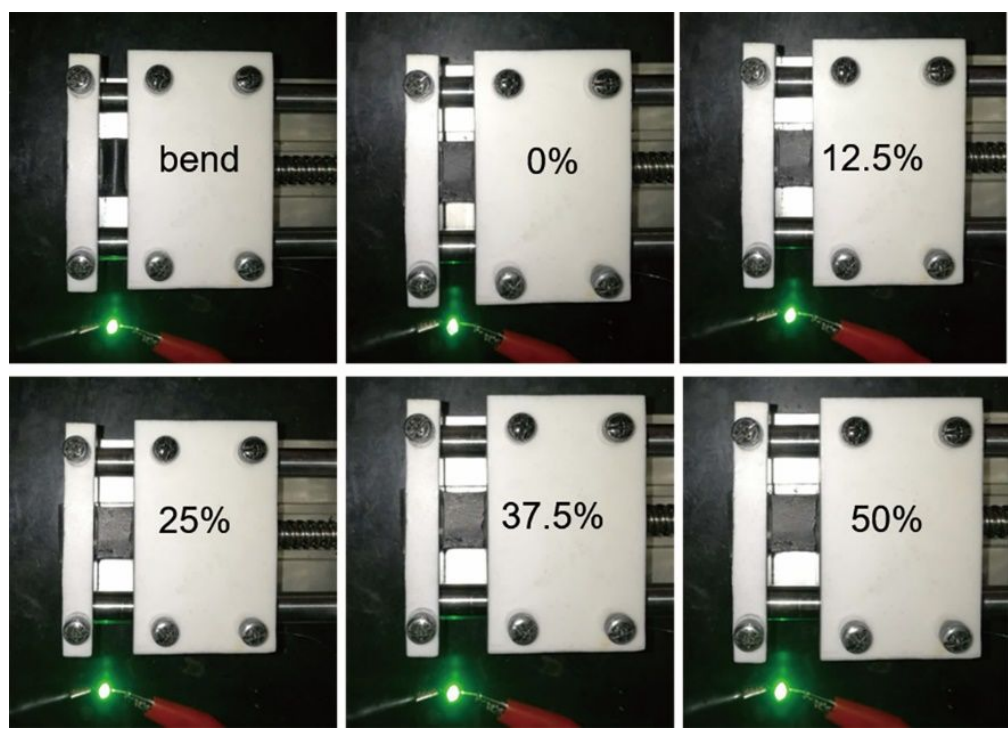

Figure S6. The photographs of an LED connected with the conductive PUE film under different deformations from $0 \%$ to $50 \%$.

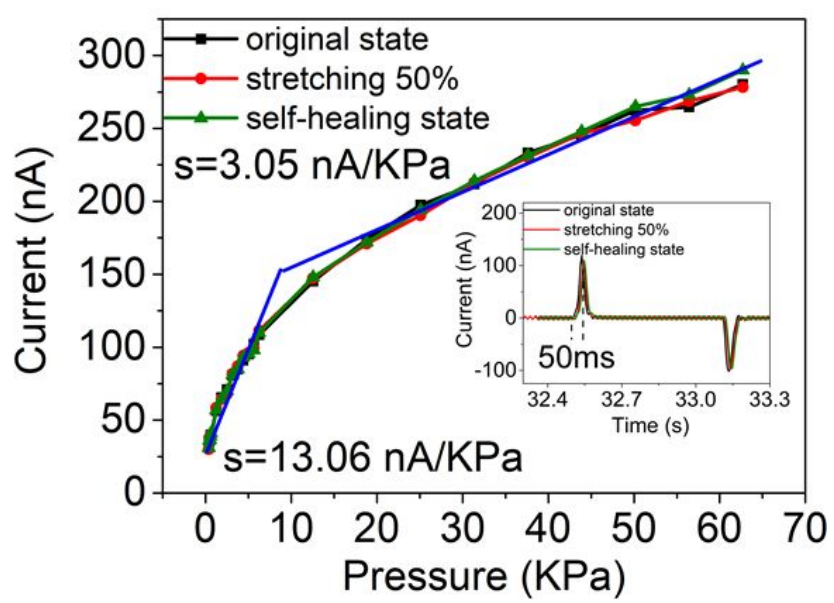

Figure S7. The sensitivity and response time of the HRSE-skin at original state, stretching state, and self-healing state. 


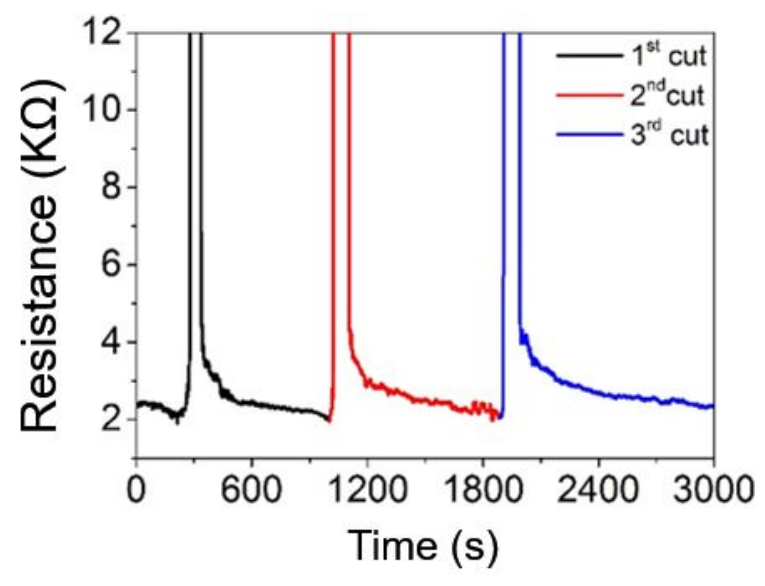

Figure S8. Self-healing of the resistance for the developed HRSE-skin after repeated cutting at the same location.

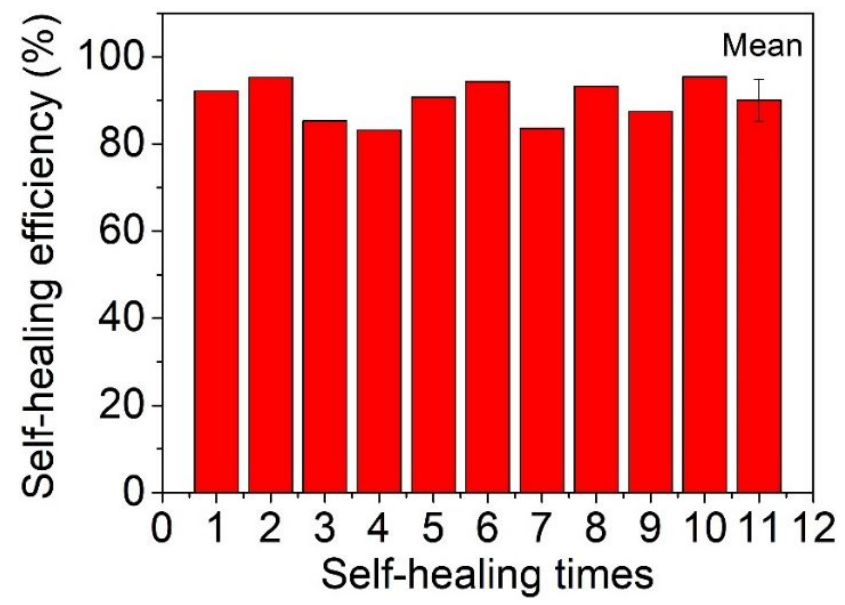

Figure S9. Self-healing efficiency of the stretchability for the developed HRSE-skin after repeated cutting at the same location.
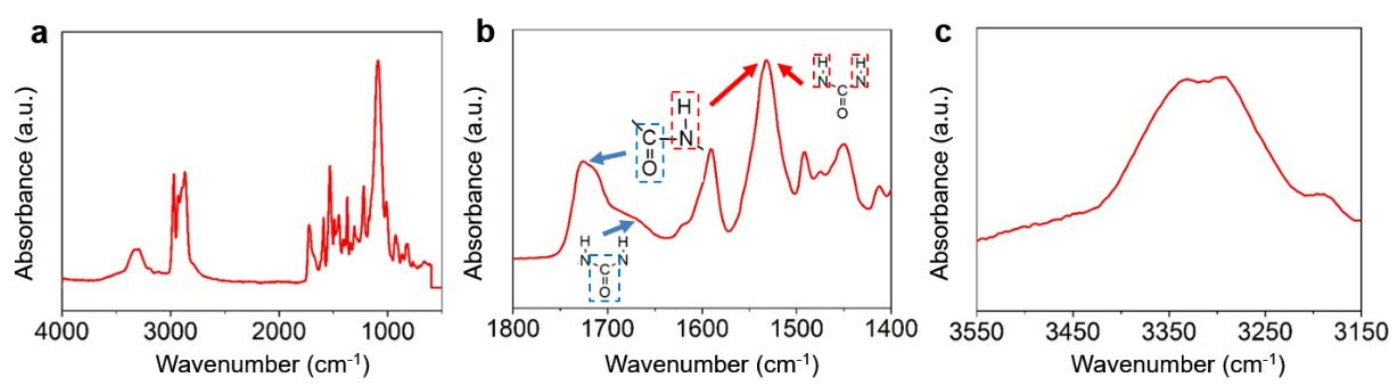

Figure S10. (a) The FTIR spectrum of the reactive mixture after curing. (b) Magnification of the FTIR spectrum recorded at $25^{\circ} \mathrm{C}$ for the PUE. The carbonyl group of urethane moiety is at $1720 \mathrm{~cm}^{-1}$ and the carbonyl group of urea is at $1670 \mathrm{~cm}^{-1}$. The 
amide group of urethane and urea is at $1534 \mathrm{~cm}^{-1}$. (c) Magnification of the N-H stretching vibration region of FTIR spectra.

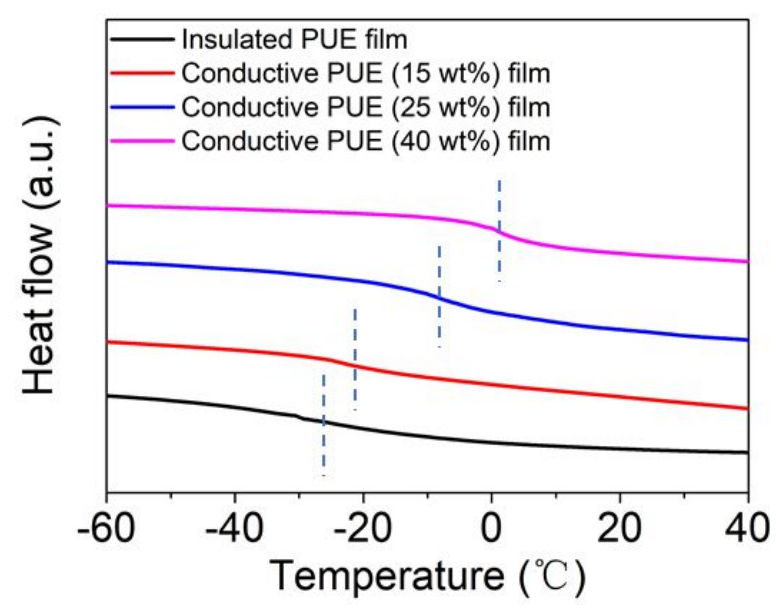

Figure S11. Differential scanning calorimetry thermal analysis of PUE with different doping content of carbon black. 